\title{
Comparison of histological and biochemical hepatic iron indexes in the diagnosis of genetic haemochromatosis
}

\author{
P M George, C Conaghan, H B Angus, T A Walmsley, B A Chapman
}

\begin{abstract}
Aims-To compare a histological hepatic iron index with a biochemical hepatic iron index, derived from atomic absorption spectroscopy measurements of hepatic iron content, for the diagnosis of genetic haemochromatosis (GH).
\end{abstract}

Methods-Histological sections of liver biopsy specimens from 70 subjects, who had previously had their biochemical hepatic iron index measured, were examined. The iron stores were scored to derive a histological hepatic iron index and were also graded from 0 to 4 by a standard grading system. The case history of each patient was then reviewed to establish a definitive clinical diagnosis and patients were classified as GH, non-GH or indeterminate.

Results-There were 26 cases of GH, 40 cases of non-GH and four indeterminate cases in whom a definite clinical diagnosis was not established. Using a biochemical hepatic iron index cut off level of $2 \cdot 0$, two cases were misclassified, with one case of GH having a biochemical hepatic iron index of 1.8 and one non-GH case having a biochemical hepatic iron index of $3 \cdot 1$. This could not have been improved by altering the cut off level. Using the recommended cut off level of $0 \cdot 15$, the histological hepatic iron index was raised in all cases of $\mathbf{G H}$, but was also increased in 11 of the 40 non-GH patients. The specificity of this histological index can be improved by increasing the cut off level to $0 \cdot 30$. A histological iron grade of $\geq 3$ is more specific than the histological index but has a lower sensitivity, which particularly affects the diagnosis of younger patients with GH.

Conclusions-The biochemical hepatic iron index is a reliable method for establishing a diagnosis of homozygous GH. In contrast, the histological hepatic iron index as originally described is non-specific and does not reliably distinguish patients with GH from others with a raised hepatic iron index due to other causes. The specificity of this index can be improved by increasing the cut off level used, but the discrimination provided by the histological index is still inferior to that provided by the biochemical hepatic iron index.

(f Clin Pathol 1996;49:159-163)

Keywords: haemochromatosis, liver biopsy.
Genetic haemochromatosis is characterised by a progressive accumulation of iron in several tissues including the liver, pancreas, heart, and skin. ${ }^{2}$ This results in tissue damage which, in the liver, can progress to fibrosis, cirrhosis and eventually hepatoma. As the complications can all be prevented by early treatment including venesection, ${ }^{3}$ accurate and early diagnosis is important. The condition is inherited in an autosomal recessive manner and is due to a gene which has been localised to chromosome 6 , close to the HLA-A gene. ${ }^{4}$ There is an increase in the frequency of HLA-A 3 in affected individuals but, although this is useful for studying the inheritance in affected families, it is not useful for diagnosis. Thus, until the gene is eventually cloned, diagnosis is dependent on the demonstration of increased iron stores. This can be done by measuring serum iron saturation and ferritin, but these parameters are non-specific. ${ }^{5}$ Liver biopsy provides the most useful laboratory method for establishing the diagnosis. ${ }^{6-8}$ In the past this relied on grading the iron stores (grades 0 to 4 ) as visualised in the histological sections. ${ }^{9}$ However, this is not really quantitative and does not incorporate the observation that iron accumulation increases with age. Thus, a biochemical hepatic iron index (BHII) was developed to overcome these problems. ${ }^{6}$ In this method hepatic iron is measured directly and the index, which reflects the rate of iron accumulation, is derived by dividing the iron content by the patient's age. More recently, a histological hepatic iron index (HHII) has been proposed. ${ }^{10}$ In this method the iron content is scored by both intensity and location and the resulting score (range 0-60) is divided by the patient's age to obtain the index. The aim of this study was to compare the use of HHII and BHII in establishing a diagnosis of genetic haemochromatosis.

\section{Methods}

Patients were selected for this study on the basis of their previous BHII results. Our laboratory analyses liver biopsy samples from throughout New Zealand but, for logistic reasons, only subjects living within the Christchurch region were considered for inclusion. Patients having liver biopsy specimens referred for BHII assessment typically were being investigated for liver dysfunction of unknown origin and had at least one raised serum iron index. All local patients who had their BHII determined between 1990 and 1994 were considered for inclusion. Those with a BHII of $2 \cdot 0$ or greater 
Table 1 Summary data for patients with and without genetic haemochromatosis*

\begin{tabular}{|c|c|c|c|c|c|c|}
\hline Patient group & $\begin{array}{l}\text { Age } \\
\text { (years) }\end{array}$ & $\begin{array}{l}\% \\
\text { Saturation }\end{array}$ & $\begin{array}{l}\text { Ferritin } \\
(\mu g / l)\end{array}$ & $B H I I$ & $H H I I$ & Grade \\
\hline $\begin{array}{l}\text { With genetic haemochromatosis }(n=26,18 \text { men }) \\
\text { Without genetic haemochromatosis }(n=40,26 \text { men })\end{array}$ & $\begin{array}{l}46 \\
45\end{array}$ & $\begin{array}{l}71 \dagger \\
30\end{array}$ & $\begin{array}{l}1828 \dagger \\
736\end{array}$ & $\begin{array}{l}8.4 \dagger \\
0.8\end{array}$ & $\begin{array}{l}0.58 \dagger \\
0.11\end{array}$ & $\begin{array}{l}3.0 \dagger \\
0.4\end{array}$ \\
\hline
\end{tabular}

were regarded as probable cases of genetic haemochromatosis and were all included. In each case the next local patient with a BHII of less than $2 \cdot 0$ was included as a control. Further controls included patients with a BHII between 1.5 and $2 \cdot 0$, irrespective of geographic location. The selected cases were then subjected to a clinical review to establish a final diagnosis (genetic haemochromatosis, not genetic haemochromatosis or indeterminate), which was based on the clinical features and laboratory information including serum ferritin concentration, iron saturation, routine histology, HLA typing, and, when available, response to venesection. Other causes of iron overload were excluded.

\section{BIOCHEMICAL IRON ASSESSMENTS}

The BHII was determined as part of the routine evaluation of these patients. The method used was as described previously. ${ }^{68}$ Briefly, the liver biopsy specimens was dried in an oven and then about $1 \mathrm{mg}$ was digested in concentrated nitric acid and the hepatic iron content was determined by air-acetylene using a Varian Spectra AA10. The BHII ( $\mu \mathrm{mol}$ iron/g dry tissue/age) was calculated. In the absence of chronic transfusion a value of 2.0 or greater is considered essentially diagnostic of genetic haemochromatosis. ${ }^{68}$

\section{HISTOLOGICAL IRON ASSESSMENTS}

Histological sections of the liver biopsy specimens from each patient were obtained and assessed retrospectively by one of us (HBA), without knowledge of the clinical or biochemical data. After staining for iron by the Perls' staining technique the HHII was de-

Table 2 Clinical characteristics of four patients classified as indeterminate for haemochromatosis

\begin{tabular}{llllllll}
\hline Patient & Sex & $\begin{array}{l}\text { Age } \\
\text { (years) }\end{array}$ & $\begin{array}{l}\% \\
\text { Saturation }\end{array}$ & $\begin{array}{l}\text { Ferritin } \\
(\mu g / l)\end{array}$ & BHII & HHII & $\begin{array}{l}\text { Histological } \\
\text { iron grade }\end{array}$ \\
\hline 1 & M & 55 & 43 & 972 & 1.8 & 0.27 & 1 \\
2 & M & 31 & 93 & 312 & 1.5 & 0.23 & 1 \\
3 & M & 50 & 31 & 628 & 1.5 & 0.38 & 2 \\
4 & M & 58 & 84 & 1120 & 1.5 & 0.23 & 2 \\
& Mean & 49 & 63 & 758 & 1.6 & 0.28 & 1.5 \\
\hline
\end{tabular}

Table 3 Performance characteristics of tests used for the diagnosis of haemochromatosis

\begin{tabular}{lllll}
\hline \multicolumn{5}{c}{ Performance characteristics } \\
\cline { 2 - 5 } Test & $\begin{array}{l}\text { Sensitivity } \\
(\%)\end{array}$ & $\begin{array}{l}\text { Specificity } \\
(\%)\end{array}$ & $\begin{array}{l}\text { Positive predictive } \\
\text { value (\%) }\end{array}$ & $\begin{array}{l}\text { Negative predictive } \\
\text { value (\%) }\end{array}$ \\
\hline \% Saturation & 82 & 89 & 82 & 89 \\
Ferritin $(\mu \mathrm{g} / \mathrm{l})$ & 92 & 52 & 61 & 89 \\
BHII & 96 & 98 & 96 & 98 \\
HHII (cut off level $=0 \cdot 15)$ & 100 & 28 & 47 & 100 \\
HHII (cut off level $=0 \cdot 30)$ & 96 & 90 & 97 & 86 \\
Grade (3 or 4) & 81 & 98 & 96 & 89 \\
\hline
\end{tabular}

rived according to the methods described by Deugnier et $a l^{10}$ : iron stores are assessed and scored numerically according to the distribution and intensity of staining with the score being weighted by the hepatocytic iron concentration. The hepatic iron score (range $0-60$ ) is divided by the patient's age to give the HHII. The biopsy specimens were also graded (from 0 to 4 ) by a standard method. ${ }^{9}$

\section{DATA ANALYSIS}

Sensitivity, specificity and the positive and negative predictive values were calculated without considering the four patients who where classified as indeterminate. Cut off levels of $\geq 60 \%$ for percentage iron saturation and of $\geq 350 \mu \mathrm{g} / 1$ (women) or $\geq 450 \mu \mathrm{g} / \mathrm{l}$ (men) for ferritin concentration were used, as these levels might alert a clinician to the possibility of a diagnosis of genetic haemochromatosis. Linear regression analysis and the statistical comparisons, using the unpaired Student's $t$ test, were performed with Microsoft EXCEL version 5.0.

\section{Results}

On the basis of the final clinical classification, there were 26 patients with genetic haemochromatosis, 40 without genetic haemochromatosis and four indeterminate cases where the clinical diagnosis was not yet certain but genetic haemochromatosis could not be excluded. There was no significant difference in the mean age or sex ratio for patients with and without genetic haemochromatosis. Table 1 summarises the patients' clinical details. The patients without genetic haemochromatosis had a range of diagnoses, including hepatitis $(n=19)$, alcoholic liver disease $(n=6)$, steatosis $(n=9)$, normal histology $(n=2)$, and one case each of steroid responsive liver disease, cholangiolitis, vitamin E deficiency, and inflammation of unknown cause. The four indeterminate cases were all male (table 2). In these four cases genetic haemochromatosis was regarded as a possible diagnosis but the presence of other factors, or of atypical serum iron studies, made the diagnosis uncertain. These patients have not been treated by venesection and are being observed to determine whether they have progressive accumulation of iron characteristic of genetic haemochromatosis.

As reported previously, ${ }^{8}$ although both serum ferritin and percentage iron saturation were higher in the patients with than in those without genetic haemochromatosis $(p<0.001)$, the serum indexes do not provide adequate discrimination between these two groups of patients (table 3). 

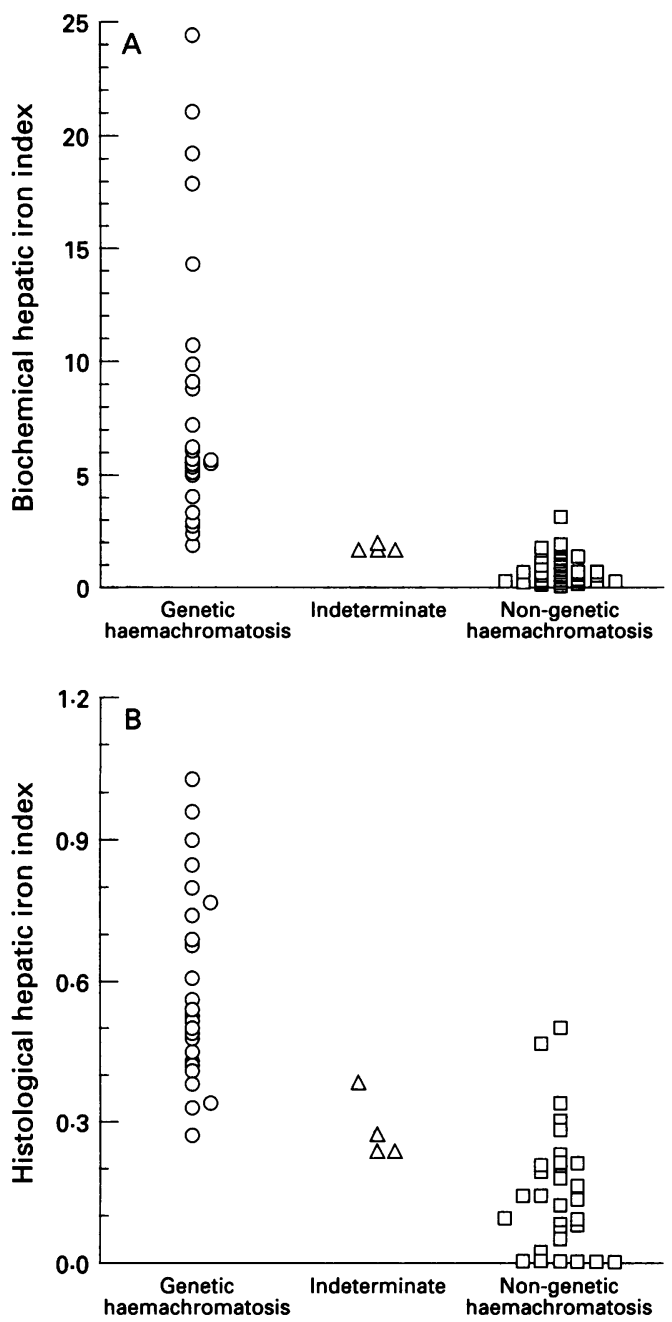

Figure 1 Hepatic iron indexes in patients with genetic haemochromatosis (O), in those without genetic haemochromatosis ( $\square$ ) and in the indeterminate cases $(\triangle) . A$, biochemical hepatic iron index; $B$, histological hepatic iron index.

The BHII values are shown in fig $1 \mathrm{~A}$. The mean values for the patients with genetic haemochromatosis, the indeterminate cases and those without genetic haemochromatosis were $8.4,1.6$ and 0.8 , respectively. Using the recommended cut off level of $2 \cdot 0,{ }^{68}$ the BHII was both sensitive (96\%) and specific (98\%), resulting in very high predictive values (table 3). Only two cases were misclassified using this index. One case, a 30 year old woman, had a BHII of 1.8 with a serum ferritin concentration of $448 \mu \mathrm{g} / \mathrm{l}$ and a percentage iron saturation of $93 \%$. Her HHII was unequivocally raised at 0.43 and the diagnosis of genetic haemo- chromatosis was confirmed by her response to quantitative venesection. In the other case the BHII of 3.1 was not consistent with either the percentage iron saturation of $4 \%$ or the HHII of 0.05 . The serum ferritin concentration was raised at $662 \mu \mathrm{g} / \mathrm{l}$ and the clinical diagnosis of chronic hepatitis caused by infection with hepatitis $C$ virus was confirmed by serology and detection of the viral RNA by polymerase chain reaction.

As shown in fig 1B, the HHII was raised in all the patients with genetic haemochromatosis; however, there was considerable overlap with values for the patients without genetic haemochromatosis and the index was raised in 11 of the latter. The mean values for patients with genetic haemochromatosis, the indeterminate cases and patients without genetic haemochromatosis were $0.58,0.28$ and 0.11 , respectively. Thus, using a cut off level of $0 \cdot 15$, as originally described, the method as applied here was sensitive but not specific (table 3 ). However, it is possible to improve the performance of the HHII by increasing the cut off level. Analysis of the sensitivity and specificity at different cut off levels indicates that a value of 0.30 provides better discrimination. At this value only one of the cases of genetic haemochromatosis would be missed and only three of the 40 patients without genetic haemochromatosis had an abnormal HHII. This results in an improved specificity of $90 \%$ with only a small reduction in sensitivity at $96 \%$. All four cases classified as indeterminate had a HHII >0.15; however, three of these cases would be in the "normal" range if the higher cut off level of 0.30 was used.

Table 4 summarises the data from the 11 patients without genetic haemochromatosis with a HHII $>0 \cdot 15$. As shown, there are a range of histological features present but alcoholic liver disease is relatively common.

As shown in fig 2, there is an overall correlation $\left(r^{2}=0.533\right)$ between BHII and HHII values in patients with and without genetic haemochromatosis.

Hepatic iron stores were also assessed using the accepted system of grading from 0 to 4 . Using this system, and accepting grades 3 and 4 as consistent with genetic haemochromatosis, we found that only one patient without genetic haemochromatosis had abnormal iron stores. However, five of the patients with genetic haemochromatosis had grade 1 (one case) or grade 2 (four cases) iron stores. These patients tended to be younger (mean age 30 years) and

Table 4 Characteristics of 11 patients without genetic haemochromatosis with a histological hepatic iron index of $>0 \cdot 15$

\begin{tabular}{|c|c|c|c|c|c|c|c|c|}
\hline $\begin{array}{l}\text { Patient } \\
\text { No. }\end{array}$ & Sex & $\begin{array}{l}\text { Age } \\
\text { (years) }\end{array}$ & $\begin{array}{l}\% \\
\text { Saturation }\end{array}$ & $\begin{array}{l}\text { Ferritin } \\
(\mu g / l)\end{array}$ & $B H I I$ & $H H I I$ & Grade & Clinical notes \\
\hline $\begin{array}{r}1 \\
2 \\
3 \\
4 \\
5 \\
6 \\
7 \\
8 \\
9 \\
10 \\
11\end{array}$ & $\begin{array}{l}M \\
M \\
F \\
M \\
M \\
M \\
F \\
F \\
M \\
M \\
M\end{array}$ & $\begin{array}{l}26 \\
57 \\
38 \\
50 \\
32 \\
61 \\
43 \\
42 \\
32 \\
49 \\
49\end{array}$ & $\begin{array}{l}21 \\
58 \\
72 \\
62 \\
31 \\
\mathrm{NA} \\
44 \\
65 \\
65 \\
40 \\
55\end{array}$ & $\begin{array}{l}330 \\
2500 \\
726 \\
2400 \\
302 \\
1096 \\
329 \\
585 \\
1635 \\
600 \\
4253\end{array}$ & $\begin{array}{l}1.7 \\
1.7 \\
0.6 \\
1.0 \\
1.8 \\
0.8 \\
1.4 \\
0.2 \\
0.9 \\
1.6 \\
0.6\end{array}$ & $\begin{array}{l}0.50 \\
0 \cdot 47 \\
0.34 \\
0 \cdot 30 \\
0 \cdot 28 \\
0 \cdot 23 \\
0 \cdot 21 \\
0 \cdot 21 \\
0 \cdot 19 \\
0 \cdot 18 \\
0 \cdot 16\end{array}$ & $\begin{array}{l}2 \\
2 \\
3 \\
2 \\
2 \\
0 \\
1 \\
1 \\
0 \\
1 \\
1\end{array}$ & $\begin{array}{l}\text { Steatosis, vitamin E deficiency, } \alpha_{1} \text {-antitrypsin deficiency, periportal iron } \\
\text { Alcoholic liver disease } \\
\text { Steatosis, focal necrosis, periportal iron ? cause } \\
\text { Alcoholic liver disease with secondary siderosis } \\
\text { Mild steatosis } \\
\text { Micronodular cirrhosis secondary to alcoholic liver disease } \\
\text { Probable heterozygous genetic haemochromatosis } \\
\text { Micronodular cirrhosis secondary to alcoholic liver disease } \\
\text { Micronodular cirrhosis, steatosis and haemosiderosis, ? alcohol } \\
\text { Steatosis with mild siderosis } \\
\text { Autoimmune chronic cholestatic liver disease }\end{array}$ \\
\hline
\end{tabular}




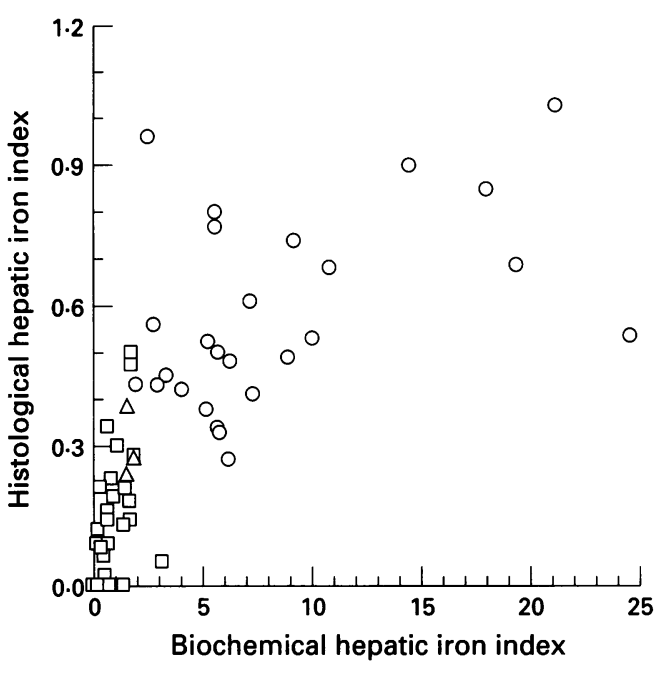

Figure 2 Correlation of the hepatic iron indexes assessed biochemically and histologically for patients with genetic haemochromatosis (O), in those without genetic haemochromatosis () and in the indeterminate cases $(\triangle)$.

included the four patients with genetic haemochromatosis who were $\leq 30$ years of age. Thus, a grade of 3 or 4 for hepatic iron stores is a specific (98\%) but relatively insensitive $(81 \%)$ test for genetic haemochromatosis, which is particularly unreliable in younger patients (table 3).

\section{Discussion}

There is increasing awareness that genetic haemochromatosis is a relatively common and treatable cause of chronic liver disease. ${ }^{11}$ This is largely due to the ready availability of serum iron studies which reflect the total body iron stores and to the recognition that carriers constitute 1 in 10 to 20 of the population. ${ }^{11}$ However, early diagnosis of genetic haemochromatosis is essential if treatment is to prevent complications due to end organ damage. ${ }^{3}$

Until the gene is cloned, and the pathogenic mutation(s) identified, diagnosis must be based on a clinical assessment in combination with serum iron studies and measurement of hepatic iron stores. Although serum ferritin was consistently raised in our cases of genetic haemochromatosis, it is non-specific and was also elevated in $55 \%$ of the patients without genetic haemochromatosis. The percentage iron saturation gives somewhat better discrimination, with increased levels being found in only $16 \%$ of the patients without genetic haemochromatosis. Thus, although useful for screening and the initial evaluation of iron stores, these tests are not reliable for the diagnosis of genetic haemochromatosis.

The BHII provides a reliable tool for establishing the diagnosis of genetic haemochromatosis and a value of $\geq 2 \cdot 0$ is essentially diagnostic. ${ }^{68}$ This is confirmed by the results of this study which show that a positive test has a very high predictive value of $96 \%$, with a negative predictive value of $98 \%$. The method is calibrated and can be controlled with $\mathrm{Na}$ tional Bureau of Standards calibrated bovine liver. However, the method is technically demanding, unsuitable for smaller laboratories, and requires collection of a larger liver biopsy specimen than is required for histology alone. Unfortunately, standard histological iron grading is an insensitive approach which does not allow for the patient's age. Thus, the report from Deugnier et al of an age corrected histological hepatic iron index ${ }^{10}$ offered a welcome approach that could be used as part of the routine histological evaluation of liver biopsy specimens. We have found that, using the recommended cut off level of $0 \cdot 15$, the HHII misclassifies almost $25 \%$ of patients without genetic haemochromatosis. We also found that a variety of other conditions give a raised HHII. This is in contrast to the original report which showed that the HHII reliably distinguished patients homozygous for genetic haemochromatosis from their heterozygous relatives. Apparently, this cannot be extended to the normal clinical situation where the problem is to distinguish patients with genetic haemochromatosis from those with other conditions. As the HHII method combines both the intensity and localisation of iron deposition with the patient's age, it might be expected to give a reliable guide to diagnosis. In practice, this is confounded by variation in the extent of iron deposition, even in adjacent lobules. Thus, scoring adjacent regions within a single biopsy specimen may give quite different scores and a subjective element is introduced in assessing the "average" pattern. This problem also exists in the standard grading methods but any variation can be documented in the text of the biopsy specimen report. Finally, the methodology is limited by Deugnier et al's failure to describe the microscopic power to be used in assessing the iron stores. In a personal communication Deugnier stated that he used a $\times 200$ magnification and therefore this magnification was used for this study. The scores in our patients with genetic haemochromatosis are comparable with those reported by Deugnier et al. ${ }^{10}$ It is therefore interesting that we found that increasing the cut off level to 0.30 resulted in improved discrimination between patients with and without genetic haemochromatosis.

In contrast, the BHII is readily standardised and we have shown good agreement with the Brisbane laboratory $\left(r^{2}=0.988\right) .{ }^{6}$ Although we used a cut off level of $2 \cdot 0$, we have previously reported that in patients with a BHII in the borderline range between 1.5 and 2.0 a diagnosis of genetic haemochromatosis cannot be automatically excluded. These patients should be followed clinically until a clear diagnosis is established. ${ }^{8}$ In this study 10 patients with a BHII in this range were deliberately included to test the methodolgy. Despite this, the BHII reliably discriminated between patients with and without genetic haemochromatosis. Only one of these patients was misclassified as having genetic haemochromatosis and four others had an indeterminate diagnosis. Using a cut off level of 0.30 the HHII correctly identified the patient with genetic haemochromatosis but misclassified the two patients without genetic 
haemochromatosis who had HHIIs of 0.47 and 0.50 . The BHII would also misclassify the one patient without genetic haemochromatosis who had an index of 3.1. This patient had an inflammatory reaction of unknown cause. The biopsy specimen had a very low dry weight of $0.22 \mathrm{mg}$ (average for other biopsy specimens was $1.4 \mathrm{mg}$ ) and so the increased BHII may reflect either contamination during processing of the specimen, analytical problems associated with the additional errors introduced for very small samples, or perhaps the presence of nonstaining iron in association with the inflammation. Although we have not quantitated these problems, it is probably undesirable to measure hepatic iron content in samples under $0.5 \mathrm{mg}$ dry weight.

Four subjects in whom a clear diagnosis could not be established were classified as indeterminate and included in this study. These patients all had HHIIs $>0.15$ but only one exceeded $0 \cdot 30$. Similarly, all of these patients had BHIIs between 1.5 and 1.8 , in the "borderline" range. Thus, continuing clinical follow up of these individuals will be important in establishing appropriate cut off levels for both methods.

A histological iron grade of 3 or 4 is a specific indicator of genetic haemochromatosis (table 3). However, grades 0 to 2 iron stores were found in five of the patients with genetic haemochromatosis. These patients were significantly younger than the other patients with genetic haemochromatosis and included all of those patients who were $\leq 30$ years old at diagnosis. In these young patients, therefore, calculation of the HHII is likely to be particularly helpful. Consistent with our finding that the iron grade is more specific than HHII (at any cut off level), 10 of the 11 patients without genetic haemochromatosis with a HHII $>0 \cdot 15$ and three of the four patients without genetic haemochromatosis with a HHII $\geq 30$ had grade 0 to 2 iron stores.

Although the BHII and HHII are correlated in patients with and without genetic haemochromatosis (fig 2), the BHII provides better discrimination between these two groups of patients. The recommended cut off level of $0 \cdot 15^{10}$ does not provide a reliable diagnosis of genetic haemochromatosis. The diagnostic value of the HHII improves if the cut off level is increased to 0.30 . However, we believe that the variation in lobular staining and the time involved in the scoring procedure, especially when done only intermittently, limits the application of this method and will prevent it from being widely adopted. Laboratories involved in the diagnosis of genetic haemochromatosis should rely on direct measurement of the hepatic iron content and calculation of the BHII. This is the current gold standard.

A cut off level of 2.0 provides good discrimination but, for the few patients with a BHII in the range $1 \cdot 5-2 \cdot 0$, the diagnosis should be based on clinical criteria. When the gene for genetic haemochromatosis is cloned, assessment of hepatic iron stores should become unnecessary as confirmation of the diagnosis by direct DNA analysis will be possible.

This work was supported by the Canterbury Medical Research Foundation who provided support for the project. We thank Sue Grant for assistance in performing the hepatic iron assays.

1 Cartwright GE, Edwards CQ, Kravitz K, Skolnick M, Amos DB, Johnson A, et al. Hereditary hemochromatosis: phenotypic expression of the disease. N Engl f Med 1979; phenotypic

2 Edwards CQ, Dadone MM, Skolnick MH, Kushner JP. Hereditary hemochromatosis. Clin Haematol 1982;11:41135

3 Niederau C, Fischer R, Sonnenberg A, Stremmel W, Trampisch HJ, Strohmeyer G. Survival and causes of death in cirrhotic and noncirrhotic patients with primary hemochromatosis. N Engl f Med 1985;313:1256-62.

4 Simon M, Le-Mignon L, Fauchet R, Yaouanq J, David V, Edan G, et al. A study of 609 HLA haplotypes marking for the hemochromatosis gene: (1) Mapping of the gene near the HLA-A locus and characters required to define a heterozygous population and (2) Hypothesis concerning the underlying cause of hemochromatosis-HLA asthe underlying cause of hemochromatos.

5 Bassett ML, Halliday JW, Ferris RA, Powell LW. Diagnosis of hemochromatosis in young subjects: predictive accuracy of biochemical screening tests. Gastroenterology 1984;87: 628-33.

6 Bassett ML, Halliday JW, Powell LW. Value of hepatic iron measurements in early hemochromatosis and determination of critical iron level associated with fibrosis. Hepatology 1986;6:24-9.

7 Sallie RW, Reed WD, Shilkin KB. Confirmation of the efficacy of hepatic tissue iron index in differentiating genetic hemochromatosis from alcoholic liver disease complicated by alcoholic hemosiderosis. Gut 1991;32:207-10.

8 Chapman BA, Horton DM, Burt MJ, Romeril KR, Walmsley TA, Grant SJ, et al. Investigation of subjects with abnormal iron studies: role of the hepatic iron index. NZ Med f 1994;107:504-7.

9 MacSween RNM, Anthony PP, Scheuer PJ. In: Pathology of the liver. 2nd edn. Edinburgh: Churchill Livingstone, 1987:185.

10 Deugnier YM, Turlin B, Powell LW, Summers KM, Moirand $\mathrm{R}$, Fletcher $\mathrm{L}$, et al. Differentiation between hetand $\mathrm{R}$, Fletcher $\mathrm{L}$, et al. Differentiation between hetby means of a histological hepatic iron index: a study of by means of a histological hepatic ir
192 cases. Hepatology 1993;17:30-4.

11 Edwards CQ, Kushner JP. Screening for hemochromatosis. $N$ Engl f Med 1993;328:1616-20. 